\title{
Integration within the classroom: How to maximise effectiveness
}

Authors: Alison Harltey and Samantha McLean. Bradford School of Pharmacy, Bradford

Background: One UK School of Pharmacy designed a new curriculum launched in September 2012. Integration with the curriculum is both vertical and horizontal. Horizontal integration occurs at each stage of the programme within each 'unit' of study and this includes the co-facilitation of classroom based activities by academic colleagues of different disciplines. In a Stage 2 unit of learning, the subject of substance misuse is presented to MPharm students for the first time. In order to maximise the effectiveness of how we deliver this learning in an integrated manner within the classroom we will evaluate both the student and the academic experience.

Description of work: Academic colleagues from the pharmacological sciences, pharmaceutical sciences and pharmacy practice worked together to create a range of integrated learning resources for use by students. This included resources for independent study outside of the classroom as well as collaborative workshop-based material. Within the classroom the different disciplines worked together to cofacilitate a series of application exercise based sessions to ensure that students understood the relevance and application of scientific knowledge to pharmacy practice-based problems.

Proposed evaluation: We will analyse quantitative and qualitative data collated via student feedback questionnaires. Staff evaluation will be conducted by the completion and reflective discussion of two institutional tools; namely a 'Peer Supported Review of Teaching Practice' and a 'Team Based Learning unit review' document. Taken together these will aid academic team reflection on how to maximise subject integration and collaborative working across disciplines to better support student learning at the unit level. The findings will inform the development of a series of recommendations for the writing and delivery of integrated learning units for staff. 